\title{
ASSOCIATION OF RETINOBLASTOMA WITH MENTAL DEFECT AND OTHER PATHOLOGICAL MANIFESTATIONS*
}

\author{
BY \\ ACHILLES TAKTIKOS \\ Department of Pathology, Institute of Ophthalmology, University of London
}

THIS paper describes an interesting association of retinoblastoma with mental defect, on some occasions further complicated by other pathological manifestations, especially in the central nervous system.

As is well known, an association between abnormalities of this system and the eye, particularly the retina, occurs in many different pathological conditions, and there is very often an abnormal metabolic and genetic background.

The association of ocular pathological conditions with mental defect is also well established, and may constitute part of the clinical picture in various diseases, as, for instance, in the cerebro-retinal degenerations of different types, the heredo-familial macular degenerations, the primary degenerations of the visual retinal cells and the phakomatoses, wherein the incidence of mental defect appears to be fairly high. According to Davenport (1918) this is 8 per cent. in neurofibromatosis.

It is likely that the association of pathological conditions in the eye with primary mental defect arises from an involvement of the nervous tissue through associated genetic defects, a concept implying the potential existence of an infinite variety of associated neuropathological manifestations, and it is conceivable that in such a way malignant neoplastic growth in the neural retinal tissue may be associated with developmental abnormalites or precocious degenerative changes in the central nervous system. This is shown by a number of cases observed during a clinicopathological study of retinoblastoma carried out at the Institute of Ophthalmology.

\section{Clinical Observations}

The first two cases concerned male babies of 3 months and 6 months of age, with sporadic bilateral retinoblastoma complicated by congenital secondary glaucoma and buphthalmos. Because of the advanced stage of the pathological process in both eyes, bilateral enucleation was performed and follow-up examinations were carried out at intervals of from 3 to 6 months, during which a defective mental development was noticed. When the patients were last examined (in November, 1962, and March, 1963, respectively) after a period of survival of about 9 years, no evidence of metastasis was found but both presented a gross mental defect, severe enough to prevent them from attending a school for mentally defective children. In view of the long-term follow-up, it seems justifiable to regard these patients as now permanently cured of their ocular tumours and practically free from any of the potential complications. Their present problem, however, consists not only of the incapacity resulting from blindness but mainly of the co-existing serious mental defect. 
Three patients who had bilateral retinoblastoma and presented typical clinical signs of advanced growth in one eye and a commencing involvement of the other, were treated by enucleation of the former whilst the latter received conservative treatment with Stallard's ${ }^{60} \mathrm{Co}$ scleral applicators, and light-coagulation on one occasion, for a very small tumour. This management resulted in preserving good uniocular vision. The follow-up investigation showed that all three children are now in excellent physical health with no evidence of any complications from retinoblastoma, but they all present gross mental defects, whilst one also shows a well-marked spasticity.

Retinoblastoma was found to be associated with mental defect and congenital hydrocephalus on three occasions.

The first was a premature baby, aged 5 months, who presented typical signs of advanced retinoblastoma in the left eye and two small white tumours in the peripheral retina in the right. The left eye was excised, and Stallard's radioactive scleral applicators were applied to the right.

The second patient, a 7-month-old male, was taken to hospital for right divergent squint. On examination under anaesthesia a retinoblastoma mass was found in the right eye, accompanied by a secondary retinal detachment. Enucleation was subsequently performed, and a small growth at the posterior nasal part of the retina in the left eye was again treated conservatively with very satisfactory results.

A follow-up of 7 years of the first patient and 4 years of the second revealed no local recurrence or metastasis, but these children are now typical examples of hydrocephalic idiots.

The third patient was a 5-year-old imbecile female with bilateral retinoblastoma. One eye was excised whilst the other was treated with repeated applications of radium seeds, but these failed to control the growth of the tumour and the second eye was eventually enucleated. Pathological examination of the right eye showed invasion of the choroid and sclera by the tumour and extra-ocular extension into the orbital tissue. A year later the child developed pneumonia and died within a few days. On post mortem examination, extensive lung metastases of retinoblastoma were revealed but no neoplastic lesion was found in the central nervous system.

Worthy of particular mention is the association of retinoblastoma with mongolism, as observed in a 2-year-old child, who presented typical features of mongolism, among them Brushfield's spots in the iris, and unilateral retinoblastoma. The child is still alive 3 years after enucleation of the right eye and there are no complications with respect to the tumour, but, as would be expected, his general health is very poor, because of severe anaemia and susceptibility to respiratory infections.

The ages of these nine patients ranged from 3 months to 7 years and the follow-up period from a few months (in the fatal cases) up to 9 years. In five patients the follow-up exceeded 4 years. Death occurred in two cases through haematogenous dissemination, but post mortem examination showed no evidence of involvement of the central nervous system.

As might be anticipated, microscopical examination of these tumours revealed no particular histological features. With regard to the degree of differentiation, they presented the usual appearances seen in retinoblastomata, corresponding to various stages in the process of differentiation, from a mere tendency of the neoplastic cells to arrange themselves in rosette-like patterns to the formation of complete, well-developed rosettes.

\section{Discussion}

All these nine cases have in common the association of retinoblastoma with mental defect, a feature to which, hitherto, no attention has been given. Herm and Heath 
(1956) mention one mentally defective patient in their series, and Falls and Neal (1951) found mental defect in five out of 73 retinoblastoma patients, commenting that children with retinoblastoma may be mentally defective more often than would be expected on the basis of the known incidence of these defects.

We observed this association in nine out of 300 cases ( 3 per cent.) in our series at the Institute of Ophthalmology, an incidence which viewed in relation to the overall incidence of mental defects in children (approximately 0.3 per cent. in the age group 5 to 9 years in Great Britain) is relatively high. It is believed that this percentage would have been even higher if the assessment of the mental state in any patient, especially those of the usual retinoblastoma age, were not so difficult particularly when minor defects are involved.

In our view, the incidence of this association is not realized and its significance is not appreciated.

Impaired mental development may occur as a result of congenital or early blindness, through the absence of optical impressions and this could, of course, apply also to blindness due to bilateral retinoblastoma. It is hardly likely, however, that such an impairment would account for serious defects such as imbecility and it would not be expected to precede blindness. Moreover, it is inconceivable that mental defect should be due to blindness in unilateral cases or in those in which the less affected eye was treated conservatively and retained good vision. The mental defect should, therefore, be regarded as a primary condition.

The association of the ocular tumour not only with mental defect but also with such pathological manifestations in the central nervous system as hydrocephalus and spasticity is equally interesting and should, like the mental defect, be interpreted as primary, since in none of our patients was there any evidence suggesting secondary involvement of the central nervous system. The association of retinoblastoma and mongolism, a condition in which mental deficiency is a typical characteristic, appears to us to be of even greater importance.

In none of the above cases was any evidence found of hereditary retinoblastoma, a fact that would have been in favour of the genetic background of the association, which would be conclusively proved if the latter were observed in familial cases. However, as the incidence of hereditary retinoblastoma is exceedingly low, this is not surprising and it is impossible to predict what the incidence of this association would be if large series of familial cases of retinoblastoma were available for follow-up.

The preponderance of bilateral involvement, as is evident in this paper, may be of some particular interest, but whether it could be in any way relevant to the wellknown higher incidence of bilaterality in hereditary retinoblastoma, and therefore of any significance in this respect, it is impossible to ascertain.

The small number of cases in which these defects were seen to be associated does not exclude the possibility of an incidental association and further observations are certainly needed. In view, however, of the rarity of all these conditions, such an association cannot be unreservedly accepted and one may ask whether these manifestations may not represent neuro-ophthalmological variants of a wide, geneticallydetermined syndrome. Indeed, the almost unlimited variety of pathological manifestations to which disturbances in fundamental physico-chemical processes within the cells may give rise, and the fact that the same nervous tissue is always 
involved in these conditions, suggest that they may all have a common or related background. Furthermore, the evidence that has recently accumulated of the paramount importance of structural or functional enzymatic defects in the genesis of degenerative and, possibly, of neoplastic changes and their predominant role in the production of pathological genes, seem to add support to this concept.

Since it is generally accepted that retinoblastoma is due to mutation of genes, and since the genetic character of more and more pathological conditions, such as mongolism, is now increasingly recognized, it is tempting to assume that the association here observed may be of chromosomal origin and may derive from co-existing chromosomal-gene defects. It is also possible that the underlying cause may be an association of pathological genes or defective linked genes capable of inducing changes leading to malignant neoplastic growth in the neural retinal cells on the one hand, and to disturbances in the central nervous system on the other. Chromosomal studies may, in future, throw light upon this hypothesis.

I wish to acknowledge the encouragement and invaluable help given to me by Professor Norman Ashton, to whom I express my grateful thanks.

\section{REFERENCES}

DAVENPORT, C. B. (1918). Proc. nat. Acad. Sci., 4, 213.

FALLS, H. F., and NEeL, J. V. (1951). A.M.A. Arch. Ophthal., 46, 367.

HeRm, R. J., and Heath, P. (1956). Amer. J. Ophthal., 41, 22.

NATIONAl Society for MENTAlly HANDiCAPPED ChILDREN (1952). "Report". London. 